\title{
The enigma of the origin of life and its timing
}

\section{MINI- REVIEW}

\author{
Martin A. Line
}

Tel: +61 36226 2636. Fax: +61 36226 2642. e-mail: Martin.Line@utas.edu.au

School of Agricultural Science, University of Tasmania, Hobart, Tasmania 7001, Australia

Keywords: life origin, panspermia, last universal ancestor, last common community

\section{Overview}

The first 3.5 billion years of evolution on Earth was dominated by unicellular life, significant multicellularity being independently evolved on numerous occasions from about 1200 million years ago in Eukarya (Carroll, 2001) or earlier in Bacteria. Rather than being novel, much of recent evolution in terms of biochemistry is derived, being attributable largely to "tinkering with the available equipment, adapting existing organs to new purposes' (Nisbet \& Sleep, 2001). An example of the manner of working of 'modern' evolution is seen in the origin of some forms of fermentation in Bacteria, which was derived from respiration, which was derived from photosynthesis, which were derived from nitrogen fixation (Burke et al., 1993). Nitrogen fixation was probably present prior to the divergence of the three domains of life, and because of its complexity, was almost certainly derived from an earlier function, perhaps as a detoxyase responsible for detoxifying cyanides or other chemicals present in the early atmosphere (Fani et al., 2000). It has been suggested that nitrogen fixation arose late in the Archaean, 2.2-2.3 gigayears ago (Gya), due to decreasing abiotic production of fixed nitrogen in the atmosphere (NavarroGonzález et al., 2001), but phylogenetic evidence strongly indicates an origin earlier than 3.5 Gya.

There is an enormous leap from pre-biotic chemistry to the complexity of DNA replication, protein manufacture and biochemical pathways existing at the time of the primary divergence of life. Although progress is being made on the evolution of some structural components and biochemical pathways, there remain numerous unsolved 'chicken and egg' problems. Margulis (1996a) said, 'To go from a bacterium to people is less of a step than to go from a mixture of amino acids to that bacterium', yet accumulated evidence from the physical and biological sciences indicates that advanced life existed at a very early stage of Earth's development. While derived evolution is clearly more efficient than de novo evolution, why then did it (coupled with symbiosis) take a further 3 gigayears to arrive at plants and animals, when most of the basic, often unique, developments appear to have been established in the first 500 million years or less?
Hence the enigma: an origin of life on Earth appears highly improbable, an origin elsewhere is highly conjectural. While this conundrum has been identified in various forms for several decades, its magnitude has dramatically increased over the last five years as new constraints are placed on the timing of the primary divergence of the domains of life (Shen et al., 2001). The problem can be likened to one faced by cosmologists a few years ago, and since resolved, that the universe appeared to be younger than the oldest stars in our galaxy. The cumulative evidence suggesting an extraterrestrial origin for life on Earth is the subject of this review.

\section{Earth's history, relevant to the origin of life and its domains}

The accretion of Earth with a metal core was completed by $\sim 4.5$ Gya (Halliday, 2001). Evidence provided by uranium-lead ages of zircon crystals within sedimentary rock having a high ${ }^{18} \mathrm{O}$ abundance, characteristic of interactions between molten rock and liquid water, indicates the possible presence of a Hadean Ocean as early as 4.2-4.3 Gya (Mojzsis et al., 2001). Until about 3.8-3.9 Gya, Earth was subjected to the late heavy meteorite bombardment (LHB) (Kerr, 2000; Nisbet \& Sleep, 2001), which was of sufficient magnitude to convert all the oceans to steam, perhaps on numerous occasions. An interesting possibility is that the LHB was responsible for selecting hyperthermophilic biota (and eliminating all others), by periodically boiling the global oceans (Nisbet \& Sleep, 2001), with perhaps two lineages (and possibly their viruses) surviving the selection, which are identified today as the deepest branches in the Bacteria and Archaea. Cohen et al. (2000) suggested that the LHB may have destroyed any existing life on Earth, or may have provided precursor molecules or suitable environments for evolution. These authors concluded that either way, this catastrophic bombardment of the Earth-moon system affected the origin and evolution of life. An alternative possibility is that the LHB might instead have seeded Earth with living cells, perhaps thermophiles (Line, 1996).

Rapid oxygenation of Earth's atmosphere occurred from 
2.2-2.4 Gya, although oxygenic photosynthesis was possibly present at 3.5-3.8 Gya. The oxidation of the atmosphere and oceans is primarily attributed to the coupling of photosynthetic dissociation of water by cyanobacteria with the burial of reduced organic material, but will inevitably have contributions from the coupling of photosynthetic dissociation of water with the UV photolysis of methane, resulting in the loss of hydrogen to space (Catling et al., 2001), and with direct hydrogen release (loss to space) by cyanobacterial mats (Hoehler et al., 2001). The oxygenation of cyanobacterial microenvironments must have preceded that of the atmosphere by a considerable margin, but it cannot be associated with the emergence of eukaryotes, the deepest lineages of which are anaerobic. The advent of oxygen evolution is possibly related to the flowering of diverse lineages within the Bacteria between 2.8-3.5 Gya, although the apparent absence of comparable diversification in the Archaea/Eukarya lineages over the equivalent timespan suggests that any such relationship may be spurious.

\section{Current concepts of the origin of life leading to the first cell}

The habitat and nature of early life has been recently reviewed by Nisbet \& Sleep (2001). Briefly, there are a number of lines of evidence indicating that the cradle of life was located in a deep- or shallow-sea hydrothermal vent at elevated temperatures. Strong selection for hyperthermophiles would also have existed on early Earth as a result of episodic meteorite bombardment. In the absence of ozone, the surface of Earth was probably bathed in lethal UV radiation for more than 2 gigayears of its existence, a problem that would not apply to the hydrothermal habitat. This habitat favours the abiotic synthesis of ammonia (Brandes et al., 1998) as well as the formation of key elements of the citric acid cycle from $\mathrm{CO}$ and $\mathrm{H}_{2} \mathrm{~S}$ in the presence of metal catalysts (Cody et al., 2000). All individual reactions for the abiotic conversion of CO to peptides have now been demonstrated (Wächtershäuser, 2000).

The sequence of evolution leading to the DNA world may have begun with prebiotic chemistry in the interstellar medium, or may have been accomplished on mineral (Ferris et al.; Trevors, 1997a, 1996) or metal surfaces entirely in habitats aquatic (particularly hydrothermal) (Russell \& Hall, 1997; Nisbet, 2000; Wächtershäuser, 2000). We have made little advance on the chirality problem of why natural sugars are right handed and natural amino acids are left handed, although the discovery that vortex forces can lead to chiral selection (Ribó et al., 2001) is intriguing. Homochirality is considered essential to life, since without it genetic material could not copy itself (Cohen, 1995). The role of ribonucleotides as coenzymes in many biological reactions suggests that these were fundamental to a primitive RNA metabolism. That an RNA world preceded the DNA world is widely accepted (Orgel, 1986; Trevors, 1997b) and recently strongly supported by developments using synthetic ribozymes (Johnston et al., 2001). Remnants of an RNA ancestry of contemporary organisms can be found in their replication machinery, in components of RNA processing and in translation apparatus, while RNA is notably absent from the process of transcription, indicating that this function was the early preserve of proteins. The need to change from an RNA- to a DNA-based genome comes from the inherent instability of RNA; RNA genomes (mainly single stranded, but including some double-stranded) are now found only in viruses, which require very few genes. Evidence is emerging of viral origins at a very early stage, perhaps prior to the divergence of the three domains (Balter, 2000), and quite possibly extant RNA viruses are remnants of the RNA world. The discovery of retroviruses, which are capable of copying RNA to DNA counterparts, provides a strong lead as to how a DNA takeover may have occurred.

Proteins perform catalysis and DNA stores information, while RNA can do both. For this reason ribozymes, RNA elements having catalytic function, have been proposed as the primeval genetic material (Joyce et al., 1987). Naturally occurring ribozymes include groups that excise introns and others that cleave RNA. While no natural ribozyme is known that can utilize nucleoside triphosphates as substrates for polymerization, considerable advances have been made using synthetic ribozymes (Szostak et al., 2001), to the point where an RNA polymerase has been constructed that can make complementary copies of any other RNA molecule up to 14 nucleotides long (Johnston et al., 2001). A single RNA sequence has also been found to be capable of adopting two different structures, each with distinct catalytic abilities, and the capacity for smooth evolutionary pathways between them has also been observed (Joyce, 2000; Schultes \& Bartel, 2000). However, significant RNA extension remains a complex process, and quite possibly the original replicator molecule may have been a simpler form of molecule, such as peptide nucleic acid or helical peptides (Piccirilli, 1995; Lee et al., 1996).

\section{The significance of the ribosome}

The present-day ribosome comprises a complex mix of RNA and over 50 proteins. It is of ancient construction, certainly preceding the divergence of the three domains and we are close to understanding the nature of its structure and function at unprecedented resolution (Yusupov et al., 2001). The ribosome has been described, together with its accessories, as probably the most sophisticated machine ever made (Garrett, 1999). In a similar vein, Dahlberg (2001) described our new view of the ribosome as "like uncovering an ancient drawing, depicting in exquisite detail a universal mechanism for one of the original steps in the evolution of life'. This analogy was intended to describe its function of translation, but could also have alluded to a deeper significance, since the progenitor ribozyme that became 
the ribosome might have been the first replicating molecule of life, to later pick up quite a different function (Nisbet \& Sleep, 2001). There are hints of a possible RNA polymerase past for the ribosome: it has a site for processing single-stranded RNA (mRNA) and three other sites for binding tRNA which are universally conserved across the domains. Also, like DNA polymerase, it has a proofreading capacity for fidelity of translation based on codon-anticodon interaction (Ogle et al., 2001).

\section{From the first cell to the last common community (LCC)}

The protocell may have consisted of a self-replicating vesicle and RNA replicase (Szostak et al., 2001). Pyrite $\left(\mathrm{FeS}_{2}\right)$ formation from $\mathrm{H}_{2} \mathrm{~S}$ and ferrous ions is a favoured first energy source for life (Wächtershäuser, 1988), these substrates being abundant in the hydrothermal vent habitat. Iron monosulfide enclosures capable of supporting a protonmotive force are also favoured in this habitat (Russell \& Hall, 1997). Alternatively, the primordial enclosure may have been the familiar lipid bilayer, since fatty acids such as oleate form micelles at high $\mathrm{pH}$ and bilayer membrane vesicles at intermediate $\mathrm{pH}$, vesicle division being facilitated by shear or thermodynamic forces (Szostak et al., 2001).

Koch (2000) has posited that stable diversity only arose after extensive evolution to gain the major attributes of cellular processes, with competitive exclusion dominating earlier stages of evolution. The primary divergence of the domains is possibly attributable to independent and different solutions to the problem of osmotic stress as cells became more complex, and the development of methanogenesis (Koch, 1994). Solutions to the problem of osmotic stress were the development of the peptidoglycan cell wall in Bacteria and of the internal cytoskeleton in the Eukarya/Archaea branch (Koch, 1994, 2000). The development of the peptidoglycan cell wall at the time of the divergence of the three domains was noted to be complex, involving the development or variation of at least seven separate but linked processes. Major endosymbiotic events between Bacteria and proto-Archaea/Eukarya (Margulis, 1996b; Gupta, 1997), coupled with concomitant development of the cytoskeleton led to the emergence of Eukarya.

Our concept of the primary division of the domains of life has shifted somewhat from the last universal (or common) ancestor to one of a LCC, relieving the 'universal ancestor' of what would otherwise be an embarrassment of biochemical attributes. The vision of a gene-sharing community of organisms at the fork of the universal tree does not negate the occurrence at some point, of a seminal mutation in a single organism leading eventually to the appearance of distinct but intertwining branches. Lateral gene transfer by transformation (Demanèche et al., 2001), transduction (Balter, 2000) or conjugation (Trevors, 1995; Ghigo, 2001) has played a key role in bacterial evolution (Ochman et al., 2000) and a limited degree of domain-crossing events has con- tinued long after their divergence. A notable example of lateral gene transfer is provided by Thermoplasma acidophilum which acquired about $17 \%$ of its proteincoding sequences from Sulfolobus solfataricus on the opposite branch of Archaea, with a further $17 \%$ apparently being acquired from representatives of the Bacteria, such as Thiobacillus (Ruepp et al., 2000).

\section{Characteristics and timing of early life}

Recent evidence has lent support to the claim of Fenchel \& Finlay (1995) that 'all basic types of bioenergetic processes probably existed 3.5 billion years ago and the biogeochemical cycling of carbon, nitrogen and sulfur was established as we know it today ...'. The canonical genetic code was undoubtedly already highly evolved prior to the divergence of the LCC and has changed little since, being at, or very close to, the optimum for error minimization (Freeland et al., 2000). There is good evidence, provided by extant relatives lying deep in the Bacteria and Archaea, that the LCC was thermophilic to extremely thermophilic and included in its repertoire the machinery for nitrogen fixation and possibly aerobic respiration (Saraste, 1994), although the utility of aerobic respiration prior to the emergence of oxygenic cyanobacteria is questionable. The presence of molybdenum, iron and sulfur in the nitrogenase enzyme complex suggests a hydrothermal heritage (Nisbet \& Sleep, 2001), and one that was almost certainly anoxic since the complex is rapidly and irreversibly inactivated in the presence of oxygen.

The ribulose-1,5-bisphosphate carboxylase/oxygenase enzyme ${ }^{13 / 12} \mathrm{C}$ signature, which is indicative of carbon dioxide fixation and possibly oxygenic photosynthesis, appears in sedimentary carbonates dating from 3.5 Gya and perhaps even before 3.7-3.8 Gya (Rosing, 1999; Nisbet \& Sleep, 2001). This isotopic evidence is supported by fossil records (although controversial) of possible cyanobacteria dating to 3.3-3.5 Gya (Des Marais, 2000), with firm palaeontological evidence of cyanobacteria dating from $2 \cdot 7$ Gya (Buick, 1992). Characteristic biomarkers of both cyanobacteria and eukaryotes appear in 2.7-gigayear-old shales (Brocks et al., 1999), and since the major lineages of anaerobic photosynthetic bacteria arose before the development of (cyanobacterial) oxygenic photosynthesis (Xiong et al., 2000), their evolution by about $2 \cdot 8-3 \cdot 0$ Gya, or perhaps much earlier, is suggested (Des Marais, 2000). The deepest calibration of a node in Bacteria is provided by the isotopic signature (depletion in ${ }^{34} \mathrm{~S}$ ) for sulfate reduction in sedimentary sulfides dated to about 3.47 Gya (Shen et al., 2001). This report provided evidence that the original precipitates were formed at temperatures below $60^{\circ} \mathrm{C}$, which is indicative of mesophiles to moderate thermophiles, suggesting that divergence of the responsible biota occurred later than that of the hyperthermophiles which constitute deeper levels of the phylogeny.

The combined evidence, particularly derived from isotopic data, places the advent of the LCC earlier than 
3.5 Gya. Synthesis of isotopic evidence with phylogenetic data (16S rDNA sequencing), extends this to probably earlier than $3 \cdot 8$ Gya. In view of the complexity of the LCC relative to that resulting from all subsequent evolution, it is difficult to avoid the conclusion that at least as great a timespan preceded the LCC as that which followed it. If so, we must search beyond Earth for the cradle of life.

\section{Possible origin of life within the outer solar system}

The recent focus has been on the transport of microbes within rock fragments following collision of a meteorite with a planet or moon: Mars being rated as a strong contender by Nisbet \& Sleep (2001). These authors also noted that it was $\sim 10^{6}$ times easier to exchange impact debris between the inner planets than between the inner planets and outer planet satellites. However, this should not be considered as an obstacle to a seeding event from beyond Mars; life when present tends to be abundant, as would splash-debris during the LHB. A low probability of occurrence in one area can be readily overcome by a high incidence of events in another.

The most important reason for invoking an extraterrestrial origin for life, probably in a hydrothermal habitat, is that such an origin provides a greater timespan for early evolution than has been available on Earth. Without this compelling reason any such theory would be superfluous. Also necessary would be the prolonged provision of liquid water, preferably coupled with stable hydrothermal vents. In our solar system this habitat will have existed on a number of the outer planets or moons, heat being provided by long-term radioactive elements of uranium, thorium and potassium, supplemented in some cases by tidal forces (e.g. Ganymede, Io, Europa and Triton). Sizeable oceans would be maintained by thermal insulation provided by water-ice blanketing on, for example, Pluto and the larger moons of Jupiter, Saturn and Neptune, nitrogenice blanketing on Triton, and atmospheric blanketing on Neptune, Pluto and Triton (Weissman et al., 1998). The size of a planet or moon is also critical to achieve chemical fractionation to maintain internal heating, eliminating the smaller icy bodies that litter the outer solar system from consideration in the present context, although they may have provided important organic chemicals to the primordial process (Anders, 1989). Some Jovian and Saturnian satellites such Ganymede, Io, Triton, and possibly Enceladus and Europa are thought to have undergone periods of active geology within a million years of their existence up to the present time. Recent volcanic eruptions and shallow flooding of water-ice appear probable for Ganymede (Schenk et al., 2001), and both Ganymede and Europa are indicated to have oceans beneath an ice surface (McCord et al., 2001).

The critical question then is one of timing. In the case of Mars, accretion was completed 4.54 Gya (Halliday, 2001), and the planet is thought to have been host to abundant liquid water from before 3.8 Gya (Newsom, 1996). Following the disappearance of the oceans, pockets of liquid water would have survived in subsurface hydrothermal plumes for prolonged periods. The most recent episodes of water-related activity on Mars resulting in landscape change, seem to have occurred within the past 10 million years (Baker, 2001). However, the evidence for liquid water on Earth at 4.2-4.3 Gya (Halliday, 2001) places severe constraints on the 'additional time' available for evolution on Mars, to only perhaps 100 million years. Application of Occam's razor thus takes the lustre off a Martian origin, there being little need for such a scenario apart from the provision of additional surface area (over that provided by Earth) for the critical event to have occurred. This argument also applies elsewhere in the solar system; the earliest significant bodies to differentiate being asteroids at 4.56 Gya (Halliday, 2001), indicating the provision of only about 200-300 million years for the generation of life on any outer planet or moon before Earth became habitable. Even if Earth was periodically subjected to sterilizing conditions by the LHB until $3.8 \mathrm{Gya}$, the additional time provided by a cradle in the outer solar system still amounts to only $\sim 600$ million years.

\section{Possible origin of life within the galaxy}

Panspermia is applied to the possible dispersion of life throughout the galaxy. Early hypotheses based on the acceleration provided to micro-organisms by solar radiation (radiopanspermia) suffered from the realization that UV light would rapidly kill the organisms being transported. Lithopanspermia overcomes this difficulty by including the possibility of microbial shielding provided by silicon or carbon coating following expulsion from a planet or moon by meteoritic impact or volcanic activity (Parsons, 1996). In such a way, microbes could survive UV radiation and still be accelerated to escape velocity from the host solar system. Promising sources of the intense radiation needed to accelerate organisms to escape velocity are red giant stars, these being relatively cool and having little ultraviolet content. Potential problems relating to the survival of bacteria during ejection from the mother planet and that of excessive speed on re-entry into Earth's atmosphere (Weber \& Mayo Greenberg, 1985) have also been answered. Good evidence exists that rocks can be splashed from Mars to Earth with minimal shocking (Melosh, 1993) and with subsurface heating of no more than $40^{\circ} \mathrm{C}$ (Weiss et al., 2000), while carboncoated bacteria have been estimated to be able to penetrate the Sun's radiation from beyond the solar system to reach Earth's atmosphere at speeds of less than $50 \mathrm{~km} \mathrm{~s}^{-1}$. Carbon/silicon shielding is considered sufficient to protect a cell for about 1 million years, in which time it could have travelled 20 light years; in this way life could propagate through the galaxy, from one solar system to the next (Parsons, 1996). It has also been noted that even if dead bacteria arrived on a habitable Earth, their nucleic acid could provide templates for subsequent evolution. 
Directed panspermia describes the deliberate seeding of life on Earth by intelligent beings: a realistic concept since we have the capacity of sending spacecraft to nearby stars with a travel time of a million years or so. This timespan would however restrict our choice of biotic travellers to microbes, but could include thermophiles similar to representatives of the LCC.

Imperative to the sustainability of panspermia hypotheses (or even of solar-system counterparts) is the prolonged survival of dormant bacterial cells. We have clear evidence for the survival of Bacillus spores in Egyptian tombs for 3500 years and the survival of various bacteria in ice for perhaps 500000 years. More recently, work has focused on amber, with reported isolations of a range of bacteria from 25-40-millionyear-old (Cano \& Borucki, 1995; Lambert et al., 1998) and from 120-million-year-old material (Greenblatt et al., 1999). The claim that viable bacteria have survived for 250 million years in salt crystals (Vreeland et al., 2000) has been questioned though not discounted (Hazen \& Roedder, 2001).

\section{Conclusions and future directions}

Evidence is accumulating that life originated at high temperature and pressure, probably in a hydrothermal vent habitat. The finding of Ribó et al. (2001) that vortex motion can lead to chiral selection should stimulate research on this aspect in relation to the formation of key amino acids and sugars in the hydrothermal context, where sustained vortexes are readily envisaged. Work on synthetic ribozymes is at an exciting stage: at the present pace of progress we might soon be able to simulate all essential facets of the RNA world. That the ribosome is a ribozyme hints of its deeper heritage; the discovery of an RNA polymerase potential within the core sequence of rRNA would provide an important 'missing link' between the RNA world and the DNA world which followed, and possibly a window to the original replicator molecule.

If Earth was the cradle for life, the time interval between its origin and the existence of the LCC appears incomprehensibly short. In view of the apparent complexity of the LCC, particularly in terms of biochemistry, it would be reasonable to allow perhaps 4 gigayears for its evolution from the primordial cell. Acceptance of such an extended period of evolution must however lead to the conclusion of an extra-terrestrial origin for life on Earth. Unfortunately, examination of other planets or moons of our solar system as potential cradles for life provides very little comfort since the time extension thereby obtained is minimal. The remaining possibility for consideration is an origin beyond our solar system via directed or undirected panspermia. The following significant constraints would tend to minimize the likelihood of directed (relative to undirected) panspermia being the option that applied to Earth.

1. Our failure to detect non-natural radio signals indicates intelligent life to be at most rare in the galaxy.
This contrasts with the potential for lithopanspermia, since planetary systems are demonstrably common and clearly undergo a lot of splashing.

2. If Earth was deliberately seeded with extra-terrestrial bacteria it took $\sim 4$ gigayears of further evolution to produce a lifeform capable of sending a spacecraft to another star. Adding a 'comfortable' 8 gigayears for the evolution from a primordial cell to the human equivalent elsewhere, takes us to the first generation of stars in our galaxy. Therefore only a very small fraction of the habitable galaxy would be seeded in this manner to the present time, again contrasting with undirected panspermia which in the scenario described would have had a 4 gigayear lead-time for galactic colonization.

3. If Earth was deliberately seeded, it is odd that it was only with microbes. Although the transport of macroorganisms such as ourselves may never be possible over interstellar distances, we foresee the time when robust, intelligent, self-replicating machines will be capable of a trip taking a million years or more. The time-span between our capacity for achieving directed panspermia using microbes (now) to that when we might provide the seed for both life and machine-intelligence is probably less than 200 years, a very small time-span in galactic terms.

Against undirected panspermia is the very low density of stars in our region of the galaxy, militating against a random serendipitous seeding event at the very time when Earth became habitable. This apparent difficulty may however relate to our poor comprehension of the capacity of meteoritic splashing to propel large numbers of organisms into space. What number of carbon/ silicon-coated microbes splattered randomly into space is needed to provide a good probability of seeding another planetary system in our part of the galaxy, and why should this number not be achievable?

Further work is needed to support contentions of the survival of bacteria for a million years or more, since this has a direct bearing on the viability of panspermia hypotheses. Particularly important is the confirmation of estimates of the survivability of ensheathed bacterial spores (and their nucleic acid) following exposure to high-energy radiation equivalent to that existing in the interstellar medium over prolonged periods. While asserting that the total energy input of energetic particles is generally orders of magnitude lower than that of UV light, Weber \& Mayo Greenberg (1985) did not consider survival of cocooned cells protected from this source.

Future expeditions to the outer solar system should shed some light on the reality or otherwise of extra-terrestrial origins of life. If the conclusions outlined here are correct, Mars and the habitable moons of other planets would have been seeded from beyond our solar system in the same event that seeded Earth. Tidal or other forces on ice crusts of some of the outer moon systems would ensure that microbes seeded at the surface would reach a warm ocean beneath. Consequently all such life would be related to that on Earth, linking to the 'base' 
of the universal phylogeny, quite possibly at a common node (point in time) regardless of its planetary or satellite origin. In that case we would certainly obtain a fascinating perspective of independent evolution from about 4 Gya, but would gain no deeper insight as to what came before.

\section{Note added in proof}

The prospects of interstellar panspermia have been dealt a blow at a recent conference on Lunar and Planetary Science (Johnson Space Centre), from calculations by planetary physicist, Jay Melosh. Simulations of the orbits of Martian rocks ejected from the solare system by Jupiter indicated a very low probability of rock capture by another stellar system, of about one meteorite ejected from a planet belonging to out solar system each 100 million years. Further calculation of the chances of rocks captured by another star hitting a terrestrial planet reduced the odds even further. A similar conclusion, that the chances of life getting to our solar system in this way were 'essentially nil', was expressed by geophysicist Norman Sleep (Kerr, 2001).

The concept of interstellar panspermia has been a philosophical luxury; it may soon become a necessity if constraints of evolutionary theory continue to conspire against an origin of life in our solar system. Perhaps fresh calculations are in order, to consider possibilities other than those provided by a Mars/Jupiter slingshot. Pluto may once have hosted a stable ocean beneath an ice cover, to therefore be a contender as a cradle for life. Would it be possible for splashed material from Pluto (by Oort cloud debris) to be expelled from the solar system by its moon Charon if the obliquity of the Pluto/Charon system was better aligned in the plane of the solar system? What of a mud/micorbial 'fog' created in a splash event being accelerated to escape velocity by a red giant? What optimal system might we construe to improve the odds of interstellar transport?

\section{References}

Anders, E. (1989). Pre-biotic organic matter from comets and asteroids. Nature 342, 255-257.

Baker, V. R. (2001). Water and the Martian landscape. Nature 412, 228-236.

Balter, M. (2000). Evolution on life's fringes. Science 289, 1866-1867.

Brandes, J. A., Boctor, N. Z., Cody, G. D., Cooper, B. A., Hazen, R. M. \& Yoder, H. S., Jr (1998). Abiotic nitrogen reduction on the early Earth. Nature 395, 365-367.

Brocks, J. J., Logan, G. A., Buick, R. \& Summons, R. E. (1999). Archean molecular fossils and the early rise of Eukaryotes. Science 285, 1033-1036.

Buick, R. (1992). The antiquity of oxygenic photosynthesis: evidence from stromatolites in sulphate-deficient Archaean lakes. Science 255, 74-77.

Burke, D. H., Hearst, J. E. \& Sodow, A. (1993). Early origin of photosynthesis: clues from nitrogenase and chlorophyll iron proteins. Proc Natl Acad Sci US A 90, 7134-7238.
Cano, R. J. \& Borucki, M. (1995). Revival and identification of bacterial spores in 25- to 40-million-year-old Dominican amber. Science 268, 1060-1064.

Carroll, S. B. (2001). Chance and necessity: the evolution of morphological complexity and diversity. Nature 409, 1102-1109.

Catling, D. C., Zahnle, K. J. \& McKay, C. P. (2001). Biogenic methane, hydrogen escape, and the irreversible oxidation of early Earth. Science 293, 839-843.

Cody, G. D., Boctor, N. Z., Filley, T. R., Hazen, R. M., Scott, J. H., Sharma, A. \& Yoder, H. S., Jr (2000). Primordial carbonylated iron-sulfur compounds and the synthesis of pyruvate. Science 289, 1337-1340.

Cohen, J. (1995). Getting all turned around over the origins of life on Earth. Science 267, 1265-1266.

Cohen, B. A., Swindle, T. D. \& Kring, D. A. (2000). Support for the lunar cataclysm hypothesis from lunar meteorite impact melt ages. Science 290, 1754-1756.

Dahlberg, A. E. (2001). The ribosome in action. Science 292, 868-869.

Demanèche, S., Bertolla, F., Buret, F., Nalin, R., Sailland, A., Auriol, P., Vogel, T. M. \& Simonet, P. (2001). Laboratory-scale evidence for lightning-mediated gene transfer in soil. Appl Environ Microbiol 67, 3440-3444.

Des Marais, D. J. (2000). When did photosynthesis emerge on Earth? Science 289, 1703-1705.

Fani, R., Gallo, R. \& Liò, P. (2000). Molecular evolution of nitrogen fixation: the evolutionary history of the nifD, nifK, nifE, and nif $\mathrm{N}$ genes. J Mol Evol 51, 1-11.

Fenchel, T. \& Finlay, B. J. (1995). Anaerobic environments. In Ecology and Evolution in Anoxic Worlds. pp. 1-31. Edited by R. M. May \& P. H. Harvey. Oxford: Oxford University Press.

Ferris, J. P., Hill, A. R., Jr, Liu, R. \& Orgel, L. E. (1996). Synthesis of long prebiotic oligomers on mineral surfaces. Nature 381, 59-61.

Freeland, S. J., Knight, R. D., Landweber, L. F. \& Hurst, L. D. (2000). Early fixation of an optimal genetic code. Mol Biol Evol 17, 511-518.

Garrett, R. (1999). Mechanics of the ribosome. Nature 400, 811-812.

Ghigo, J.-M. (2001). Natural conjugative plasmids induce bacterial biofilm development. Nature 412, 442-445.

Greenblatt, C. L., Davis, A., Clement, B. G., Kitts, C. L., Cox, T. \& Cano, R. J. (1999). Diversity of microorganisms isolated from amber. Microb Ecol 38, 58-68.

Gupta, R. S. (1997). Protein phylogenies and signature sequences: evolutionary relationships within prokaryotes and between prokaryotes and eukaryotes. Antonie Leeuwenhoek 72, 49-61.

Halliday, A. N. (2001). In the beginning ... Nature 409, 144-145. Hazen, R. M. \& Roedder, E. (2001). How old are bacteria from the Permian age? Nature 411, 155.

Hoehler, T. M., Bebout, B. M. \& Des Marias, D. J. (2001). The role of microbial mats in the production of reduced gases on the early Earth. Nature 412, 324-327.

Johnston, W. K., Unrau, P. J., Lawrence, M. S., Glasner, M. E. \& Bartel, D. P. (2001). RNA-catalysed RNA polymerization: accurate and general RNA-templated primer extension. Science 292, 1319-1325.

Joyce, G. F. (2000). Ribozyme evolution at the crossroads. Science 289, 401-402.

Joyce, G. F., Schwartz, A. W., Miller, S. L. \& Orgel, L. E. (1987). The case for an ancestral genetic system involving simple 
analogues of the nucleotides. Proc Natl Acad Sci USA 84, 4398-4402.

Kerr, R. A. (2000). Beating up on a young Earth, and possibly life. Science 290, 1677.

Kerr, R. A. (2001). Rethinking water on Mars and the origin of life. Science 292, 39-40.

Koch, A. L. (1994). Development and diversification of the last universal ancestor. J Theor Biol 168, 269-280.

Koch, A. L. (2000). The bacterium's way for safe enlargement and division. Appl Environ Microbiol 66, 3657-3663.

Lambert, L. H., Cox, T., Mitchell, K., Rosselló-Mora, R. A., DelCueto, C., Dodge, D. E., Orkland, P. \& Cano, R. J. (1998). Staphylococcus succinus sp. nov., isolated from Dominican amber. Int J Syst Bacteriol 48, 511-518.

Lee, D. H., Granja, J. R., Martinez, J. A., Severin, K. \& Reza Ghadiri, (1996). A self-replicating peptide. Nature 382, 525-528.

Line, M. A. (1996). In the beginning... Todays Life Sci 8, 18-22.

McCord, T. B., Hansen, G. B. \& Hibbitts, C. A. (2001). Hydrated salt minerals on Ganymede's surface: evidence of an ocean below. Science 292, 1523-1525.

Margulis, L. (1996a). Quoted in Horgan, J.The End of Science, chapter 5, The end of evolutionary biology, pp. 140-141. Reading, MA: Addison-Wesley Publishing.

Margulis, L. (1996b). Archaeal-eubacterial mergers in the origin of Eukarya: phylogenetic classification of life. Proc Natl Acad Sci U S A 93, 1071-1076.

Melosh, H. J. (1993). Blasting rocks off planets. Nature 363, 498-499.

Mojzsis, S. J., Harrison, T. M. \& Pidgeon, R. T. (2001). Oxygenisotopic evidence from ancient zircons for liquid water at the Earth's surface 4,300 Myr ago. Nature 409, 178-181.

Navarro-González, R., McKay, C. P. \& Nna Mvondo, D. (2001). A possible nitrogen crisis for Archaean life due to reduced nitrogen fixation by lightning. Nature 412, 61-64.

Newsom, H. E. (1996). Martians in a deep freeze. Nature 379, 205-206.

Nisbet, E. (2000). The realms of Archaean life. Nature 405, 625-626.

Nisbet, E. G. \& Sleep, N. H. (2001). The habitat and nature of early life. Nature 409, 1083-1091.

Ochman, H., Lawrence, J. G. \& Groisman, E. A. (2000). Lateral gene transfer and the nature of bacterial innovation. Nature 405, 299-304.

Ogle, J. M., Brodersen, D. E., Clemons, W. M., Jr, Tarry, M. J., Carter, A. P. \& Ramakrishnan, V. (2001). Recognition of cognate transfer RNA by the 30S ribosomal subunit. Science 292, 897-902.

Orgel, L. E. (1986). RNA catalysis and the origins of life. J Theor Biol 123, 127-149.

Parsons, P. (1996). Dusting off panspermia. Nature 383, 221-222. Piccirilli, J. A. (1995). RNA seeks its maker. Nature 376, 548-549.
Ribó, J. M., Crusats, J., Sagués, F., Claret, J. \& Rubires, R. (2001). Chiral sign induction by vortices during the formation of mesophases in stirred solutions. Science 292, 2063-2066.

Rosing, M. T. (1999). ${ }^{13} \mathrm{C}$-depleted carbon microparticles in $>$ $3700-\mathrm{Ma}$ sea-floor sedimentary rocks from West Greenland. Science 283, 674-676.

Ruepp, A., Graml, W., Santos-Martinez, M. L. \& 7 other authors (2000). The genome sequence of the thermoacidophilic scavenger Thermoplasma acidophilum. Nature 407, 508-513.

Russell, M. J. \& Hall, A. J. (1997). The emergence of life from iron monosulphide bubbles at a submarine hydrothermal redox and pH front. J Geol Soc London 154, 377-402.

Saraste, M. (1994). Structure and evolution of cytochrome oxidase. Antonie Leeuwenhoek 65, 285-287.

Schenk, P. M., McKinnon, W. B., Gwynn, D. \& Moore, J. M. (2001). Flooding of Ganymede's bright terraines by low-viscosity waterice lavas. Nature 410, 57-60.

Schultes, E. A. \& Bartel, D. P. (2000). One sequence, two ribozymes: implications for the emergence of new ribozyme folds. Science 289, 448-452.

Shen, Y., Buick, R. \& Canfield, D. E. (2001). Isotopic evidence for microbial sulphate reduction in the early Archaean era. Nature $410,77-81$.

Szostak, J. W., Bartel, D. P. \& Luigi Luisi, P. (2001). Synthesising life. Nature 409, 387-390.

Trevors, J. T. (1995). Molecular evolution in bacteria. Antonie Leeuwenhoek 67, 315-324.

Trevors, J. T. (1997a). Bacterial evolution and silicon. Antonie Leeuwenhoek 71, 271-276.

Trevors, J. T. (1997b). Evolution of bacterial genomes. Antonie Leeuwenhoek 71, 265-270.

Vreeland, R. H., Rosenzweig, W. D. \& Powers, D. (2000). Isolation of a 250 million-year-old halotolerant bacterium from a primary salt crystal. Nature 407, 897-900.

Wächtershäuser, G. (1988). Pyrite formation, the first energy source for life: a hypothesis. Syst Appl Microbiol 10, 207-210.

Wächtershäuser, G. (2000). Life as we don't know it. Science 289, 1307-1308.

Weber, P. \& Mayo Greenberg, J. (1985). Can spores survive in interstellar space? Nature 316, 403-407.

Weiss, B. P., Kirschvink, J. L., Baudenbacher, F. J., Vali, H., Peters, N. T. Macdonald, F. A. \& Wikswo, J. P. (2000). A low temperature transfer of ALH84001 from Mars to Earth. Science 290, 791-795.

Weissman, P. R., McFadden, L. A. \& Johnson, T. V. (editors) (1998). Encyclopedia of the Solar System, New York: Academic Press.

Xiong, J., Fischer, W. M., Inoue, K., Nakahara, M. \& Bauer, C. E. (2000). Molecular evidence for the early evolution of photosynthesis. Science 289, 1724-1730.

Yusupov, M. M., Yusupova, G. Zh., Baucom, A., Lieberman, K., Earnest, T. N., Cate, J. H. D. \& Noller, H. F. (2001). Crystal structure of the ribosome at $5.5 \AA$ resolution. Science 292, 883-896. 